Z. Wahrscheinlichkeitstheorie verw. Gebiete $68,503-519(1985)$
Zeitschrift für

Wahrscheinlichkeitstheorie und verwandte Gebiete

(C) Springer-Verlag 1985

\title{
Change-of-Variance Sensitivities in Regression Analysis
}

\author{
Elvezio Ronchetti and Peter J. Rousseeuw \\ Department of Statistics, Princeton University, Princeton, New Jersey 08544, USA \\ Department of Mathematics, Delft University of Technology, \\ Julianalaan 132, $2628 \mathrm{BL}$ Delft, The Netherlands
}

\begin{abstract}
Summary. The change-of-variance function is defined for estimators of regression coefficients. Both an unstandardized and a standardized form of the change-of-variance sensitivity are introduced, and their relation with the corresponding gross-error-sensitivities is investigated. The problems of optimal robustness lead to the Hampel-Krasker and the Krasker-Welsch estimators. At the same time, also the scale parameter has to be estimated robustly. By means of the change-of-variance sensitivity, optimal robust redescending scale estimators are constructed.
\end{abstract}

\section{Introduction}

The linear regression model is used very often in statistical analysis. It is wellknown that the classical least squares estimator is sensitive to departures from the normality assumption on the errors. In fact, this procedure loses efficiency very rapidly when the distribution of the errors becomes slightly nonnormal, as is often the case (Hampel 1973, Huber 1973). Many robust procedures have been proposed in the last years. For robust estimation of regression coefficients see, for instance, Huber (1973), Bickel (1975), Holland and Welsch (1977), Hampel (1978), Ronchetti (1979), Ruppert and Carroll (1980), Ronchetti and Rousseeuw (1980), Krasker and Welsch (1982), Siegel (1982), Donoho and Huber (1983) and Rousseeuw (1982b). New robust tests for linear models have been developed by Schrader and McKean (1977), Schrader and Hettmansperger (1980) and Ronchetti (1982a,b).

In this paper we investigate the robustness properties of a wide class of regression estimators generalizing least squares. We follow the infinitesimal approach to robustness, which is based on the central notions of influence function and change-of-variance function.

The influence function (IF) and the corresponding gross-error-sensitivity have been defined by Hampel (1974) for estimators. Essentially, the IF is the first derivative of an estimator viewed as a functional, and it describes the 
normalized influence of contaminated data on the estimator. Later, this concept was extended to tests (Rousseeuw and Ronchetti 1979, 1981; Lambert 1981).

The change-of-variance function (CVF) can be viewed as a second order measure for robustness, and describes the infinitesimal stability of the asymptotic variance of an estimator. This concept was discovered in 1972 by Hampel, who used it to construct the so-called hyperbolic tangent estimators of 1ocation. The function as well as the estimators were referred to briefly in (Hampel 1973, p. 98 and 1974, p. 393). Later, these ideas were developed more rigorously in the location case by Rousseeuw (1981, 1982a) and Hampel, Rousseeuw and Ronchetti (1981).

In this work we extend the latter concept to regression, including the estimation of a scale parameter. Both an unstandardized and a standardized form of the change-of-variance sensitivity are introduced, and their relation with the corresponding gross-error-sensitivities is investigated. We then construct most robust and optimally robust estimators with respect to the influence function and the change-of-variance function.

\section{2. $M$-estimators for the Regression Model}

In this paper the following linear regression model is considered. Denote by $H_{\theta, \sigma}$ the distribution of the iid observations $\left(x_{i}, y_{i}\right), 1 \leqq i \leqq n$, in case

$$
y_{i}=x_{i}^{t} \theta+e_{i}
$$

where the $x_{i} \in \mathbb{R}^{p}$ have the distribution $K, y_{i} \in \mathbb{R}, \theta \in \mathbb{R}^{p}, \sigma>0$, and $e_{i}$ is independent of $x_{i}$ and distributed according to $\Phi(e / \sigma)$ where $\Phi$ denotes the standard normal cumulative function.

It is well-known that the standard least squares estimator for the parameters $\theta$ and $\sigma$ is not robust. One way to cope with this problem is to study a large class of estimators generalizing least squares, and to select more robust procedures in that class. It appears that $M$-estimators (from "generalized maximum likelihood", see Huber 1964) are most appropriate for this purpose. In this paper, attention is focused on the formulation of Maronna and Yohai (1981), where $M$-estimators $\left(T_{n}, S_{n}\right)$ of $(\theta, \sigma)$ are defined as simultaneous solutions of

$$
\begin{gathered}
\sum_{i=1}^{n} \eta\left(x_{i},\left(y_{i}-x_{i}^{t} T_{n}\right) / S_{n}\right) x_{i}=0 \\
\sum_{i=1}^{n} \chi\left(\left(y_{i}-x_{i}^{t} T_{n}\right) / S_{n}\right)=0
\end{gathered}
$$

where $\eta: \mathbb{R}^{p} \times \mathbb{R} \rightarrow \mathbb{R}$ and $\chi: \mathbb{R} \rightarrow \mathbb{R}$. The solution $\left(T_{n}, S_{n}\right)$ can also be written as $\left(T\left(H_{n}\right), S\left(H_{n}\right)\right)$, where $H_{n}$ is the empirical distribution and the couple $(T, S)$ of functionals is given by

$$
\begin{gathered}
\int \eta\left(x,\left(y-x^{t} T(\tilde{H})\right) / S(\tilde{H})\right) d \tilde{H}(x, y)=0 \\
\int \chi\left(\left(y-x^{t} T(\tilde{H})\right) / S(\tilde{H})\right) d \tilde{H}(x, y)=0
\end{gathered}
$$

for any distribution $\tilde{H}$ of $(x, y)$ on $\mathbb{R}^{p} \times \mathbb{R}$. 
The best known case is what we shall call "Huber-type regression" (1973, 1981), where

$$
\eta(x, y)=\psi(y)
$$

for some function $\psi$. (It generalizes least squares, corresponding to $\eta_{L S}(x, y)$ $=y$.) This form of robust regression limits the effect of errors in the $y_{i}$, but should be used with caution if also outhers in the $x_{i}$ can occur. To overcome this difficulty, Mallows (1975) proposed to use

$$
\eta(x, y)=w(x) \psi(y)
$$

where $w$ is a weight function. On the other hand, Schweppe introduced functions of the type

$$
\eta(x, y)=w(x) \psi(y / w(x))
$$

(Handschin et al. 1975, Hill 1977). For a comparison of (2.5), (2.6) and (2.7), see Krasker and Welsch (1982, Sect. 2) and Maronna and Yohai (1981, Sect. 4). In practice, these estimates can be calculated by means of the ROBETH library, which is being developed by Marazzi (1980).

Maronna and Yohai (1981, Sect. 4) show that $T_{n}$ and $S_{n}$ are asymptotically independent, provided $\eta$ and $\chi$ are sufficiently regular. That is, the asymptotic behaviour of $T_{n}$ only depends on $\eta$, and that of $S_{n}$ only depends on $\chi$. Therefore, we shall first deal with robust estimation of the regression parameter $\theta$ using (2.2) as if $\sigma=1$ were already known, and postpone (2.3) to Sect. 4.

\section{Estimation of the Regression Coefficients}

The regression coefficients are the components of the vector parameter $\theta$ in (2.1), and can be estimated by means of (2.2). In this section we assume $\sigma=1$ is known. The central model is $H=H_{0,1}$, for which $\theta=0$ and $\sigma=1$. This implies that $H$ equals the product measure $K \otimes \Phi$, where $\Phi$ is the standard normal. We restrict our attention to the class $\mathscr{T}$ of all functions $\eta: \mathbb{R}^{p} \times \mathbb{R} \rightarrow \mathbb{R}$ satisfying

(i) For all $x, \eta(x, \cdot)$ is well-defined and continuous for all $y$ not belonging to the finite set $C(x, \eta)$. In each point of $C(x, \eta), \eta(x, \cdot)$ has finite left and right limits which are different. Also $\eta(x,-y)=-\eta(x, y)$ and $\eta(x, y) \geqq 0$ for positive $y$;

(ii) For all $x$, the set $D(x, \eta)$ of points in which $\eta(x, \cdot)$ is continuous but in which $\eta^{\prime}(x, y):=\frac{\partial}{\partial y} \eta(x, y)$ is not defined or not continuous, is finite;

(iii) $Q(\eta, H):=E_{H}\left[\eta^{2}(x, y) x x^{t}\right]$ is nonsingular;

(iv) $M(\eta, H):=E_{H}\left[\eta^{\prime}(x, y) x x^{t}\right]=E_{H}\left[y \eta(x, y) x x^{t}\right]$ is nonsingular.

The skew-symmetry of $\eta$ (condition $i$ ) implies that the functional $T$ of (2.4) is Fisher-consistent (Hampel 1974, p. 390), which means that $T\left(H_{\theta, 1}\right)=\theta$ for all $\theta$. Two functions $\eta_{1}$ and $\eta_{2}$ are said to be equivalent when $\eta_{1}=s \eta_{2}$ for some constant $s>0$.

Maronna and Yohai (1981) showed the consistency of $T_{n}$ and the multivariate asymptotic normality of $n^{1 / 2}\left(T_{n}-\theta\right)$, with asymptotic mean zero and 
asymptotic covariance matrix

$$
V(\eta, H)=M^{-1}(\eta, H) Q(\eta, H) M^{-1}(\eta, H)
$$

by using some additional moment conditions on $H$. Note that

$$
M S E(\eta, H):=\operatorname{tr} V(\eta, H)
$$

is the asymptotic mean squared error of $T_{n}$ at $H$.

\section{3a. Unstandardized Sensitivities}

From now on, we only consider the functional $T=T(H)$. Let us look at the contaminated distribution $H_{(x, y), \varepsilon}=(1-\varepsilon) H+\varepsilon \Delta_{(x, y)}$ where $0<\varepsilon<1$ and $\Delta_{(x, y)}$ is the probability measure which puts all its mass at $(x, y)$. It describes a mixture which contains a fraction $\varepsilon$ of outliers at $(x, y)$.

Definition $I$ (Hampel 1974, 1978). The influence function (IF) of $T$ at $H$ is defined as the $p \times 1$ vector

$$
\operatorname{IF}(x, y ; T, H)=\frac{\partial}{\partial \varepsilon}\left[T\left(H_{(x, y), \varepsilon}\right)\right]_{\varepsilon=0}
$$

in those $x$ and $y$ where it exists. The unstandardized gross-error-sensitivity of $T$ (at $H$ ) is

$$
\gamma_{u}^{*}(\eta)=\sup \left\{\|\operatorname{IF}(x, y ; T, H)\| ; x \in \mathbb{R}^{p}, y \notin C(x, \eta)\right\}
$$

where $\|\ldots\|$ denotes the usual Euclidean norm in $\mathbb{R}^{p}$.

Calculation yields IF $(x, y ; T, H)=\eta(x, y) M^{-1}(\eta, H) x$, so

$$
\gamma_{u}^{*}(\eta)=\sup _{x, y \neq C(x, \eta)}|\eta(x, y)| \cdot\left\|M^{-1}(\eta, H) x\right\| .
$$

This $\gamma_{u}^{*}$ describes the worst possible influence of contamination by outliers on the asymptotic value of $T_{n}$. Note that the mean squared error at $H$ is bounded by this sensitivity, because

$$
\operatorname{MSE}(\eta, H) \leqq\left(\gamma_{u}^{*}(\eta)\right)^{2}
$$

making use of (3.1) to (3.3). On the other hand, it is also possible to investigate the infinitesimal stability of (3.1):

Definition 2. The change-of-variance function (CVF) of $T$ at $H$ is defined as the $p \times p$ matrix

$$
\operatorname{CVF}(x, y ; T, H)=\frac{\partial}{\partial \varepsilon}\left[V\left(\eta, H_{(x, y), \varepsilon}\right)\right]_{\varepsilon=0}
$$

in those $x$ and $y$ where it exists. The unstandardized change-of-variance sensitivity of $T$ (at $H$ ) is

$$
\kappa_{u}^{*}(\eta)=\sup \left\{\operatorname{trCVF}(x, y ; T, H) / \operatorname{tr} V(\eta, H) ; x \in \mathbb{R}^{p}, y \notin D(x, \eta)\right\} .
$$


Definition 2 generalizes the change-of-variance sensitivity of the one-dimensional location case, corresponding to $p=1$ and $x \equiv 1$ (Rousseeuw 1981, Hampel, Rousseeuw and Ronchetti 1981). In this case, the CVF describes the infinitesimal stability of the asymptotic variance $V(\psi, F)$ in the vicinity of the model distribution $\Phi$. Therefore, $\kappa^{*}$ gives the largest possible slope of $\ln V(\psi, F)$ in the direction of contaminated distributions. From this, we can approximate the maximal asymptotic variance over a gross-error-neighborhood $\mathscr{P}_{\varepsilon}$ (in the sense of Huber 1964) by $V(\psi, \Phi) \exp \left(\varepsilon \cdot \kappa^{*}(\psi)\right)$, which turns out to be surprisingly adequate even up to $\varepsilon=10 \%$. This also explains why Huber's minimax asymptotic variance theory and the change-of-variance sensitivity lead to very similar optimal estimators. In the case of unrestricted $M$-estimators, both approaches yield the Huber estimator (Huber 1964, Rousseeuw 1981), and for redescending estimators one obtains almost identical tanh-estimators (Collins 1976, Hampel et al. 1981).

The calculation of the CVF in regression yields:

$$
\begin{aligned}
\operatorname{CVF}(x, y ; T, H)= & M^{-1} Q M^{-1}+\eta^{2}(x, y) M^{-1} x x^{t} M^{-1} \\
& -\eta^{\prime}(x, y)\left[M^{-1} x x^{t} M^{-1} Q M^{-1}+M^{-1} Q M^{-1} x x^{t} M^{-1}\right]
\end{aligned}
$$

where $M=M(\eta, H)$ and $Q=Q(\eta, H)$. Hence,

$$
\begin{aligned}
\mathcal{K}_{u}^{*}(\eta)= & \sup _{x, y \notin D(x, \eta)}\left[1+\eta^{2}(x, y)\left\|M^{-1} x\right\|^{2} / \operatorname{tr} V(\eta, H)\right. \\
& \left.-2 \eta^{\prime}(x, y)\left(M^{-1} x\right)^{t} Q M^{-1}\left(M^{-1} x\right) / \operatorname{tr} V(\eta, H)\right] .
\end{aligned}
$$

Note that for $y$ in $C(x, \eta)$ we obtain a Dirac delta function in $-2 \eta^{\prime}(x, y)$, which is multiplied by the (not necessarily positive) quadratic form $\left(M^{-1} x\right)^{t} Q M^{-1}\left(M^{-1} x\right)$. If this delta function (with all its factors) is negative in (3.6), then it does not contribute to $\kappa_{u}^{*}(\eta)$; if it is positive, then $\kappa_{u}^{*}(\eta)=\infty$. At $H_{\theta, \sigma}$ one should replace $y$ by $\left(y-x^{t} \theta\right) / \sigma$ in (3.3) and (3.6), but this does not change anything.

We say that the regression estimator $T$ is $B_{u}-$ robust when $\gamma_{u}^{*}(\eta)$ is finite, and we call it $V_{u}$-robust when $\kappa_{u}^{*}(\eta)$ is finite.

Theorem 1. $V_{u}$-robustness implies $B_{u}$-robustness. In fact, $\gamma_{u}^{*}(\eta) \leqq\left[\left(\kappa_{u}^{*}(\eta)\right.\right.$ $-1) \operatorname{MSE}(\eta, H)]^{1 / 2}$. If $\eta$ is nondecreasing in $y$ and $Q M^{-1} \geqq 0$, then $V_{u}$-robustness and $B_{u}$-robustness are equivalent and $\kappa_{u}^{*}(\eta)=1+\gamma_{u}^{*}(\eta)^{2} / \operatorname{MSE}(\eta, H)$.

All proofs can be found in the Appendix. The notation $Q M^{-1} \geqq 0$ means that $z^{t} Q M^{-1} z \geqq 0$ for all vectors $z$. For example, for least squares regression $\left(\eta_{L S}(x, y)=y\right)$ it holds that $Q M^{-1}=I_{p} \geqq 0$, where $I_{p}$ is the $p \times p$ unit matrix. We find $\gamma_{u}^{*}\left(\eta_{L S}\right)=\infty$ and $\kappa_{u}^{*}\left(\eta_{L S}\right)=\infty$ so least squares regression is neither $B_{u^{-}}$ robust nor $V_{u}$-robust, which illustrates Theorem 1.

Theorems 2 and 3 will provide an example which is both $B_{u}$-robust and $V_{u}$ robust. However, there exist $B_{u}$-robust estimators which are not $V_{u}$-robust (Rousseeuw 1981, Sect. 2). Basically, such estimators have influence functions which are bounded but contain discontinuities or very steep slopes, causing local instabilities of the asymptotic covariance at nearby distributions. 
Theorem 2. Always $\gamma_{u}^{*}(\eta) \geqq p(\pi / 2)^{1 / 2} / E[\|x\|]$. If $E\left[x x^{t} /\|x\|\right]$ is a scalar matrix, then $\eta(x, y)=\operatorname{sign}(y) /\|x\|$ reaches this lower bound, so we call this estimator most $B_{u}$-robust.

(Here, a scalar matrix is a real multiple of the unit matrix.) When $p=1$ or $K$ is radially symmetric, i.e.

$$
K \text { depends only on }\|x\|,
$$

then $E\left[x x^{t} /\|x\|\right]$ is indeed a scalar matrix. When $p=1$ (regression line through the origin), Theorem 2 gives the most $B_{u}$-robust estimator $T_{n}=\operatorname{median}\left\{y_{i} / x_{i}\right\}$ (see Krasker 1980 , p. 1340). When also $x \equiv 1$ (location), the solution reduces to the sample median.

Theorem 2 improves Krasker's lower bound (1980, Proposition 2) which he gave for the special family of estimators we shall deal with in Theorems 4 and 5. Note that the most $B_{u}$-robust estimator of Theorem 2 has a Mallows-type $\eta$ function (2.6), but can also be considered as a member of the Schweppe class (2.7) by writing $\operatorname{sign}(y)$ as $\operatorname{sign}(y /\|x\|)$. The following result generalizes Theorem 2 of (Rousseeuw 1982 a).

Theorem 3. Always $\kappa_{u}^{*}(\eta) \geqq 2$. If $E\left[x x^{t} /\|x\|\right]$ is a scalar matrix, then $\eta(x, y)$ $=\operatorname{sign}(y) /\|x\|$ attains this lower bound, so we call this estimator most $V_{u}$-robust.

The Hampel-Krasker estimator (Krasker 1980) is given by

$$
\eta_{H K(c)}(x, y)=\psi_{c}(y\|A x\|) /\|A x\|
$$

where $\psi_{c}$ is the Huber function $\psi_{c}(z)=\min (c, \max (z,-c))$ and where the matrix $A$ has to satisfy

$$
M\left(\eta_{H K(c)}\right)=E\left[(2 \Phi(c /\|A x\|)-1) x x^{t}\right]=A^{-1} .
$$

Unfortunately, there are some problems concerning the existence of this estimator. There is a lower bound on $\mathcal{c}$ (given by Theorem 2), and Krasker (1980) shows the existence for sufficiently large $c$, but one does not know how large $c$ must be. From now on, we restrict ourselves to $p=1$ or $(R)$, in which case this problem can be solved. We shall show that the Hampel-Krasker estimators fill the gap between the most robust estimator of Theorems 2 and 3 and least squares.

Lemma 1. Assuming $p=1$ or $(R)$ holds, (3.7) exists if and only if $c>p(\pi / 2)^{1 / 2} / E[\|x\|] . \quad$ Moreover,$\quad \gamma_{u}^{*}\left(\eta_{H K(c)}\right)=c \quad$ and $\quad c \longmapsto \kappa_{u}^{*}\left(\eta_{H K(c)}\right)=1$ $+c^{2} / \operatorname{tr}\left(V\left(\eta_{H K(c)}, H\right)\right)$ is a continuous function onto $(2, \infty)$.

Theorem 4. Assume $p=1$ or $(R)$, in which case attention is restricted to functions $\eta(x, y)=\tilde{\eta}(\|x\|, y)$. For each $c>p(\pi / 2)^{1 / 2} / E[\|x\|]$ there exists $\eta_{H K(c)}$ such that $\gamma_{u}^{*}\left(\eta_{H K(c)}\right)=c$, and $\eta_{H K(c)}$ minimizes $\operatorname{MSE}(\eta, H)$ among all $\eta$ satisfying $\gamma_{u}^{*}(\eta) \leqq c$. Any other solution is equivalent to $\eta_{H K(c)}$.

This optimality result (which also holds under more general conditions, assuming $\eta_{H K(c)}$ exists) was proven by Krasker (1980, Proposition 4). A similar result had been conjectured by Hampel (1978, subsection 3.3), generalizing the optimal robustness of the Huber function in the location model (Hampel 1974). 
We shall now show that the Hampel-Krasker estimator is also optimal with respect to $\kappa_{u}^{*}$.

Theorem 5. Assume $p=1$ or $(R)$, in which case attention is restricted to functions $\eta(x, y)=\tilde{\eta}(\|x\|, y)$. For each $k>2$, there exists a function $\eta_{H K_{(c)}}$ such that $\kappa_{u}^{*}\left(\eta_{H K(c)}\right)=k$, and $\eta_{H K(c)}$ minimizes $\operatorname{MSE}(\eta, H)$ among all $\eta$ satisfying $\kappa_{u}^{*}(\eta) \leqq k$. Any other solution of this extremal problem is equivalent to $\eta_{\mathrm{HK}(c)}$.

\section{3b. Self-Standardized Sensitivities}

It was noted by Krasker and Welsch (1982) that the unstandardized grosserror-sensitivity is not invariant with respect to linear parameter transforms. Indeed, if we put $\tilde{\theta}:=B \theta$ where $B$ is a nonsingular $p \times p$ matrix, then

$$
\begin{aligned}
\tilde{M} & =B^{-t} M B^{-1} \\
\tilde{Q} & =B^{-t} Q B^{-1} \\
\gamma_{u}^{*}(\tilde{\eta}) & =\sup |\eta(x, y)| \cdot\left\|B M^{-1} x\right\|
\end{aligned}
$$

which is in general different from $\gamma_{u}^{*}(\eta)$ because the vector norm $\left\|M^{-1} x\right\|$ is not invariant. As a consequence, the Hampel-Krasker estimator (3.7) which was based on $\gamma_{u}^{*}$ is not invariant either. To overcome this lack of invariance, the following notion was introduced:

Definition $l^{\prime}$ (Krasker and Welsch 1982). The (self-)standardized gross-errorsensitivity of $T$ (at $H$ ) is defined as

$$
\gamma_{s}^{*}(\eta)=\sup \left\{\left[\operatorname{IF}(x, y ; T, H)^{t} V(\eta, H)^{-1} \operatorname{IF}(x, y ; T, H)\right]^{1 / 2} ; x \in \mathbb{R}^{p}, y \notin C(x, \eta)\right\} .
$$

Now the influence of outliers is measured with respect to the covariance matrix of the estimator itself. Krasker and Welsch (1982) and Stahel (1981) compare this standardization with some other possibilities. Calculations yields

$$
\gamma_{s}^{*}(\eta)=\sup _{x, y \notin C(x, \eta)}|\eta(x, y)|\left(x^{t} Q(\eta)^{-1} x\right)^{1 / 2}
$$

This new sensitivity is invariant, because quadratic forms like $x^{t} Q x$ are invariant. It is also possible to introduce a standardized version of $\kappa_{u}^{*}$ with this invariance property:

Definition $2^{\prime}$. The (self-) standardized change-of-variance sensitivity of $T$ (at $H$ ) is defined as

$$
\kappa_{s}^{*}(\eta):=\sup \left\{\operatorname{tr}\left(\operatorname{CVF}(x, y ; t, H) V(\eta, H)^{-1}\right) ; x \in \mathbb{R}^{p}, y \notin D(x, \eta)\right\} .
$$

Some calculations using (3.5) yield

$$
\kappa_{s}^{*}(\eta)=\sup _{x, y \notin D(x, \eta)}\left[p+\eta^{2}(x, y) x^{t} Q^{-1} x-2 \eta^{\prime}(x, y) x^{t} M^{-1} x\right]
$$

which is also invariant. Moreover, $\kappa_{s}^{*}$ is strongly related to $\gamma_{s}^{*}$, as the following theorems will show. We say that $T$ is $B_{s}$-robust when $\gamma_{s}^{*}(\eta)<\infty$, and we call it $V_{s}$-robust in case $\kappa_{s}^{*}(\eta)<\infty$. 
Remark 1. Note that Definitions 1,2,1' and $2^{\prime}$ can be applied to other types of estimation, where both sample space and parameter space may have arbitrary dimensions; it suffices to allow contamination in all points of the sample space. Hampel (1978) already considered the general $\gamma_{u}^{*}$, whereas Stahel (1981) worked with $\gamma_{s}^{*}$ in the general case. Note that $\kappa_{u}^{*}$ and $\kappa_{s}^{*}$ always coincide when the parameter space is one-dimensional, as in the simple location model, where they are simply denoted by $\kappa^{*}$ (Rousseeuw 1981).

Theorem 1'. $V_{s}$-robustness implies $B_{s}$-robustness. In fact, $\gamma_{s}^{*}(\eta) \leqq\left[\kappa_{s}^{*}(\eta)-p\right]^{1 / 2}$. If $\eta$ is nondecreasing in $y$ and $M>0$, then $V_{s}$-robustness and $B_{s}$-robustness are equivalent and $\kappa_{s}^{*}(\eta)=p+\gamma_{s}^{*}(\eta)^{2}$.

For example, for least squares $\left(\eta_{L S}(x, y)=y\right)$ both $\gamma_{s}^{*}=\infty$ and $\kappa_{s}^{*}=\infty$.

Theorem $2^{\prime}$. Always $\gamma_{s}^{*}(\eta) \geqq \sqrt{p}$. If $E\left[x x^{t} /\|x\|^{2}\right]$ is a scalar matrix, then $\eta(x, y)$ $=\operatorname{sign}(y) /\|x\|$ reaches this lower bound, so we call this estimator most $B_{\text {s-robust. }}$

If $p=1$ or $(R)$ holds, then $E\left[x x^{t} /\|x\|^{2}\right]=\frac{1}{p} I_{p}$ is a scalar matrix.

Theorem $3^{\prime}$. Always $\kappa_{s}^{*}(\eta) \geqq 2 p$. If $E\left[x x^{t} /\|x\|^{2}\right]$ is a scalar matrix, then $\eta(x, y)$ $=\operatorname{sign}(y) /\|x\|$ reaches this lower bound, so we call this estimator most $V_{s}$-robust.

The Krasker-Welsch estimator (1982) is given by

$$
\eta_{K W(c)}(x, y)=\psi_{c}(y\|A x\|) /\|A x\|
$$

where the matrix $A$ has to satisfy

$$
Q\left(\eta_{K W(c)}\right)=E\left[\psi_{c}^{2}(y\|A x\|)\|A x\|^{-2} x x^{t}\right]=\left(A^{t} A\right)^{-1} .
$$

Lemma 1'. The Krasker-Welsch estimator exists if and only if $c>\sqrt{p}$. It holds that $\gamma_{s}^{*}\left(\eta_{K W(c)}\right)=c$ and $\kappa_{s}^{*}\left(\eta_{K W(c)}\right)=p+c^{2}$, so $\kappa_{s}^{*}\left(\eta_{K W(c)}\right)$ takes all values in the interval $(2 p, \infty)$.

In order to formulate the next results we need some terminology. It is said that $\eta$ dominates $\tilde{\eta}$ if $x^{t}(V(\eta, H)-V(\tilde{\eta}, H)) x \leqq 0$ for all $x$ and there exists some $x$ for which $x^{t}(V(\eta, H)-V(\tilde{\eta}, H)) x<0$. We say that the function $\tilde{\eta}$ is admissible in a class of functions when there is no member $\eta$ in that class which dominates $\tilde{\eta}$. It turns out (Stahel 1981, p. 32) that this is weaker than saying that $\tilde{\eta}$ minimizes $\operatorname{tr} V(\eta, H)=\operatorname{MSE}(\eta, H)$.

Theorem 4' (Stahel 1981, p. 137). For each $c>\sqrt{p}$, there exists a function $\eta_{K W(c)}$ which is admissible among all $\eta$ satisfying $\gamma_{s}^{*}(\eta) \leqq c$.

In fact, Krasker and Welsch (1982) showed that whenever there exists a function minimizing the matrix $V(\eta, H)$ (in the strong sense of positive definite matrices) subject to $\gamma_{s}^{*}(\eta) \leqq c$, then it is equivalent to $\eta_{K W(c)}$.

Theorem 5'. For each $k>2 p$ there exists a function $\eta_{K W(c)}$ such that $\kappa_{s}^{*}\left(\eta_{K W(c)}\right)$ $=k$, and $\eta_{K W(c)}$ is admissible among all $\eta$ satisfying $\kappa_{s}^{*}(\eta) \leqq k$.

In Table 1 we summarize the results obtained by means of the IF and the $\mathrm{CVF}$ in the cases of estimation of a location parameter (both in general and 
Table 1. Summary of Results

a) Estimation of a location parameter (unrestricted $M$-estimators)

\begin{tabular}{|c|c|c|c|}
\hline & $\begin{array}{l}\text { Robust } \\
\text { (finite } \\
\text { sensitivity) }\end{array}$ & $\begin{array}{l}\text { Most robust } \\
\text { (minimal } \\
\text { sensitivity) }\end{array}$ & $\begin{array}{l}\text { Optimal robust } \\
\text { (sensitivity } \leqq \text { constant, } \\
\text { minimize asymp. variance) }\end{array}$ \\
\hline $\begin{array}{l}B \\
\text { (bias) } \\
\mathrm{IF}, \gamma^{*}\end{array}$ & $\begin{array}{l}V \text {-robust } \\
\text { implies } \\
B \text {-robust, }\end{array}$ & $\begin{array}{l}\text { median } \\
\text { (Rousseeuw 1982) }\end{array}$ & $\begin{array}{l}\text { Huber estimator } \\
\text { (Hampel 1974) }\end{array}$ \\
\hline $\begin{array}{l}V \\
\text { (variance) } \\
\mathrm{CVF}, \kappa^{*}\end{array}$ & $\begin{array}{l}\text { equivalent for } \\
\text { monotone } \psi \\
\text { (Rousseeuw 1981) }\end{array}$ & $\begin{array}{l}\text { median } \\
\text { (Rousseeuw 1982) }\end{array}$ & $\begin{array}{l}\text { Huber estimator } \\
\text { (Rousseeuw 1981) }\end{array}$ \\
\hline
\end{tabular}

b) Estimation of a location parameter (redescending $M$-estimators)

\begin{tabular}{|c|c|c|c|}
\hline & $\begin{array}{l}\text { Robust } \\
\text { (finite } \\
\text { sensitivity) }\end{array}$ & $\begin{array}{l}\text { Most robust } \\
\text { (minimal } \\
\text { sensitivity) }\end{array}$ & $\begin{array}{l}\text { Optimal robust } \\
\text { (sensitivity } \leqq \text { constant, } \\
\text { minimize asymp. variance) }\end{array}$ \\
\hline $\begin{array}{l}B \\
\text { (bias) } \\
\text { IF }, \gamma^{*}\end{array}$ & $\begin{array}{l}\text { all redescending } \\
\psi \text { are } B \text {-robust, }\end{array}$ & $\begin{array}{l}\text { skipped median } \\
\text { (Rousseeuw 1982) }\end{array}$ & $\begin{array}{l}\text { skipped Huber estimator } \\
\text { (Rousseeuw 1982) }\end{array}$ \\
\hline $\begin{array}{l}V \\
\text { (variance) } \\
\mathrm{CVF}, \kappa^{*}\end{array}$ & $\begin{array}{l}\text { not all } \psi \text { are } \\
\text { V-robust } \\
\text { (Rousseeuw 1981) }\end{array}$ & $\begin{array}{l}\text { median-type } \\
\text { tanh-estimator } \\
\text { (Rousseeuw 1982) }\end{array}$ & $\begin{array}{l}\text { tanh-estimator } \\
\text { (Hampel et al 1981) }\end{array}$ \\
\hline
\end{tabular}

c) Estimation of regression coefficients (unstandardized sensitivities)

\begin{tabular}{|c|c|c|c|}
\hline & $\begin{array}{l}\text { Robust } \\
\text { (finite } \\
\text { sensitivity) }\end{array}$ & $\begin{array}{l}\text { Most robust } \\
\text { (minimal } \\
\text { sensitivity) }\end{array}$ & $\begin{array}{l}\text { Optimal robust } \\
\text { (sensitivity } \leqq \text { constant, } \\
\text { minimize asymp. MSE at } H \text { ) }\end{array}$ \\
\hline $\begin{array}{l}B_{u} \\
\text { (bias) } \\
\text { IF, } \gamma_{u}^{*}\end{array}$ & $\begin{array}{l}V_{u} \text {-robust } \\
\text { implies } \\
B_{u} \text {-robust }\end{array}$ & $\begin{array}{l}\eta(x, y)=\frac{\operatorname{sign}(y)}{\|x\|} \\
\text { (Theorem 2) }\end{array}$ & $\begin{array}{l}\text { Hampel-Krasker estimator } \\
\text { (Krasker 1980) }\end{array}$ \\
\hline $\begin{array}{l}V_{u} \\
\text { (variance) } \\
\text { CVF, } \kappa_{u}^{*}\end{array}$ & $\begin{array}{l}\text { equivalent for } \\
\text { monotone } \eta \\
\text { (Theorem 1) }\end{array}$ & $\begin{array}{l}\eta(x, y)=\frac{\operatorname{sign}(y)}{\|x\|} \\
\text { (Theorem 3) }\end{array}$ & $\begin{array}{l}\text { Hampel-Krasker estimator } \\
\text { (Theorem 5) }\end{array}$ \\
\hline
\end{tabular}

d) Estimation of regression coefficients (self-standardized sensitivities)

\begin{tabular}{|c|c|c|c|}
\hline & $\begin{array}{l}\text { Robust } \\
\text { (finite } \\
\text { sensitivity) }\end{array}$ & $\begin{array}{l}\text { Most robust } \\
\text { (minimal } \\
\text { sensitivity) }\end{array}$ & $\begin{array}{l}\text { Optimal robust } \\
\text { (admissible among } \eta \text { for } \\
\text { which sensitivity } \leqq \text { const.) }\end{array}$ \\
\hline $\begin{array}{l}B_{s} \\
\text { (bias) } \\
\text { IF }, \gamma_{s}^{*}\end{array}$ & $\begin{array}{l}V_{s} \text {-robust } \\
\text { implies } \\
B_{s} \text {-robust }\end{array}$ & $\begin{array}{l}\eta(x, y)=\frac{\operatorname{sign}(y)}{\|x\|} \\
\text { (Theorem 2') }\end{array}$ & $\begin{array}{l}\text { Krasker-Welsch estimator } \\
\text { (Stahel 1981) }\end{array}$ \\
\hline $\begin{array}{l}V_{s} \\
\text { (variance) } \\
\text { CVF, } \kappa_{s}^{*}\end{array}$ & $\begin{array}{l}\text { equivalent for } \\
\text { monotone } \eta \\
\text { (Theorem } 1^{\prime} \text { ) }\end{array}$ & $\begin{array}{l}\eta(x, y)=\frac{\operatorname{sign}(y)}{\|x\|} \\
\text { (Theorem 3') }\end{array}$ & $\begin{array}{l}\text { Krasker-Welsch estimator } \\
\text { (Theorem } 5^{\prime} \text { ) }\end{array}$ \\
\hline
\end{tabular}


for redescenders) and estimation of regression coefficients (both with unstandardized and self-standardized sensitivities). For the definition of $M$-estimators of location we refer to Huber (1964), and redescending $M$-estimators are described in Hampel et al. (1981).

Remark 2. Samarov (1983, personal communication) is presently combining the IF and the CVF to approximate the mean squared error at contaminated distributions, which yields related optimality results.

\section{Estimation of the Scale Parameter}

Let us now move to the estimation of $\sigma$, assuming $\theta=0$ is known. We restrict our attention to the class $\mathscr{S}$ of all mappings $\chi$ satisfying

(i) $\chi$ is well-defined and continuous on $\mathbb{R} \backslash C(\chi)$, where $C(\chi)$ is finite. In each point of $C(\chi)$ there exist finite left and right limits of $\chi$ which are different. Also $\chi(-y)=\chi(y)$, and there exists $d>0$ such that $\chi(y) \leqq 0$ on $(0, d)$ and $\chi(y) \geqq 0$ on $(d, \infty)$;

(ii) the set $D(\chi)$ of points in which $\chi$ is continuous but in which $\chi^{\prime}$ is not defined or not continuous, is finite;

(iii) $\int \chi d \Phi=0$ and $\int \chi^{2} d \Phi<\infty$;

(iv) $0<\int y \chi^{\prime}(y) d \Phi(y)=\int\left(y^{2}-1\right) \chi(y) d \Phi(y)<\infty$;

where $\Phi$ is the standard normal distribution.

Condition (iii) implies that the functional $S$ of (2.4) is Fisher-consistent, which means that $S\left(H_{0, \sigma}\right)=\sigma$ for all $\sigma$. Two functions $\chi_{1}$ and $\chi_{2}$ are said to be equivalent when $\chi_{1}=s \chi_{2}$ for some $s>0$.

Maronna and Yohai (1981) showed the consistency of $S_{n}$ and the asymptotic normality of $n^{1 / 2}\left(S_{n}-\sigma\right)$, with asymptotic variance

$$
V_{1}(\chi, H)=\int \chi^{2}(y) d H(x, y) /\left(\int y \chi^{\prime}(y) d H(x, y)\right)^{2} .
$$

As noted in Remark 1 , we can now apply Definitions $1,2,1^{\prime}$ and $2^{\prime}$. The influence function $\operatorname{IF}(x, y ; S, H)$ equals $\chi(y) / \int y \chi^{\prime}(y) d \Phi(y)$ (see Huber 1981, p. 137), so the unstandardized gross-error-sensitivity is given by

$$
\gamma_{u}^{*}(\chi)=\sup _{x, y \notin C(\chi)}|\operatorname{IF}(x, y ; S, H)|=\sup _{x, y \notin C(x)} \frac{|\chi(y)|}{\int y \chi^{\prime}(y) d \Phi(y)},
$$

whereas the (self-)standardized gross-error sensitivity equals

$$
\gamma_{s}^{*}(\chi)=\sup _{x, y \notin C(\chi)} \frac{|\operatorname{IF}(x, y ; S, H)|}{V_{1}(\chi, H)^{1 / 2}}=\sup _{x, y \notin C(\chi)} \frac{|\chi(y)|}{\left(\int \chi^{2} d \Phi\right)^{1 / 2}} .
$$

Thus $\left(\gamma_{u}^{*} / \gamma_{s}^{*}\right)^{2}=V_{1}$, and $B_{u}$-robustness and $B_{s}$-robustness are equivalent, so we simply talk about $B$-robustness.

The change-of-variance function $\operatorname{CVF}(x, y ; S, H)$ equals $\left[1+\chi^{2}(y)\right.$. $\left.\left(\int \chi^{2} d \Phi\right)^{-1}-2 y \chi^{\prime}(y)\left(\int z \chi^{\prime}(z) d \Phi(z)\right)^{-1}\right]\left(\int \chi^{2} d \Phi\right) /\left(\int z \chi^{\prime}(z) d \Phi(z)\right)^{2}$. As the parameter space $(0, \infty)$ is one-dimensional, we note that $\kappa_{u}^{*}$ and $\kappa_{s}^{*}$ coincide. Therefore, we 
simply call this notion the change-of-variance sensitivity:

$$
\begin{aligned}
\kappa^{*}(\chi) & =\sup _{x, y \neq D(\chi)} \frac{\operatorname{CVF}(x, y ; S, H)}{V_{1}(\chi, H)} \\
& =\sup _{x, y \notin D(\chi)}\left[1+\frac{\chi^{2}(y)}{\int \chi^{2} d \Phi}-\frac{2 y \chi^{\prime}(y)}{\int z \chi^{\prime}(z) d \Phi(z)}\right] .
\end{aligned}
$$

Remark 3. Note that the expressions (4.2) to (4.4) are identical to those for the one-dimensional normal scale model. There the observations $y_{1}, \ldots, y_{n}$ are distributed according to $\Phi(y / \sigma)$, where $\sigma$ can be estimated by means of the $M$ estimator $S_{n}$ given by $\sum_{i=1}^{n} \chi\left(y_{i} / S_{n}\right)=0$.

Now it is possible to develop a theory very much like the one for location (Rousseeuw 1981, 1982a) or regression coefficients (Sect.3), where it is proven that $V$-robustness implies $B$-robustness, and where the most robust and optimal robust members of $\mathscr{S}$ in the sense of $\gamma_{u}^{*}, \gamma_{s}^{*}$ and $\kappa^{*}$ are described. However, consideration of $\gamma_{s}^{*}$ and $\kappa^{*}$ in this situation brings the same results as those already known for $\gamma_{u}^{*}$. Therefore, we do not treat these results here but refer the interested reader to Ronchetti and Rousseeuw (1982) instead.

However, it is possible to construct new estimators by means of $\kappa^{*}$ in the case where one wants to reject large outliers completely. For this purpose, we restrict our attention to redescending $M$-estimators of scale, that is, to members of the class

$$
\mathscr{S}_{r}=\{\chi \in \mathscr{F}, \chi(y)=0 \quad \text { for all }|y| \geqq r\},
$$

where $0<r<\infty$ is fixed. We shall now undertake to find the estimators $\chi_{r, k}$ in $\mathscr{S}_{r}$ which minimize $V_{1}(\chi, H)$ subject to an upper bound $k$ on the change-ofvariance sensitivity $\kappa^{*}$.

It is expected that $\chi_{r, k}$ will be parabolic in the central region in order to attain high efficiency. On the other hand, it seems like a good idea to have [1 $\left.+\chi_{r, k}^{2}(y)\left(\int \chi_{r, k}^{2} d \Phi\right)^{-1}-2 y \chi_{r, k}^{\prime}(y) \cdot\left(\int z \chi_{r, k}^{\prime}(z) d \Phi(z)\right)^{-1}\right]$ identical to $k$ on the redescending part, in order to achieve $\mathcal{K}^{*}\left(\chi_{r, k}\right)=k$. The latter equality gives us a differential equation, from which an explicit expression of $\chi_{r, k}$ can be obtained. As no downward jumps are allowed (because otherwise $\kappa^{*}=\infty$ ), we also expect that the optimal $\chi_{r, k}$ is continuous. All these heuristic arguments together suggest that $\chi_{r, k}$ should have the following form:

$$
\begin{aligned}
\chi_{r, k}(y) & =y^{2}-1+a & & 0 \leqq|y| \leqq p \\
& =\left(A_{1}(k-1)\right)^{1 / 2} \tanh \left[\frac{1}{2}\left((k-1) B_{1}^{2} / A_{1}\right)^{1 / 2} \ln \left(\frac{r}{|y|}\right)\right] & & p \leqq|y| \leqq r \\
& =0 & & |y| \geqq r
\end{aligned}
$$

where $0<p<r$ and (in order that $\chi_{r, k}$ be continuous at $p$ )

$$
p^{2}-1+a=\left(A_{1}(k-1)\right)^{1 / 2} \tanh \left[\frac{1}{2}\left((k-1) B_{1}^{2} / A_{1}\right)^{1 / 2} \ln \left(\frac{r}{p}\right)\right] .
$$


Moreover, we assume that $\chi_{r, k}$ satisfies $\int \chi_{r, k} d \Phi=0, \int \chi_{r, k}^{2} d \Phi=A_{1}$, $\int z \chi_{r, k}^{\prime}(z) d \Phi(z)=B_{1}>0$ and $k \geqq 1+(a-1)^{2} / A_{1}$. Figure 1 gives a picture of such a function $\chi_{r, k}$. It follows that $0<a<1 \quad\left(a>0\right.$ because otherwise $\int \chi_{r, k} d \Phi<\int\left(y^{2}\right.$ $-1) d \Phi(y)=0$, and $a<1$ because otherwise $\chi_{r, k}$ would be nonnegative, hence $\int \chi_{r, k} d \Phi$ would be strictly positive). The existence of $\chi_{r, k}$ can be showed by means of the same techniques as in Theorem 3.1 of (Hampel, Rousseeuw and Ronchetti 1981). The condition $k \geqq 1+(a-1)^{2} / A_{1}$ gives a lower bound on $k$. (Consider the CVF at $y=0$, where it holds that $\operatorname{CVF}(x, 0 ; S, H) / V_{1}(\chi, H)=1$ $+\chi^{2}(0) / A_{1}=1+(a-1)^{2} / A_{1}$. If smaller values of $k$ were considered, then $\left.\kappa^{*}(\chi) \geqq 1+(a-1)^{2} / A_{1}>k.\right)$

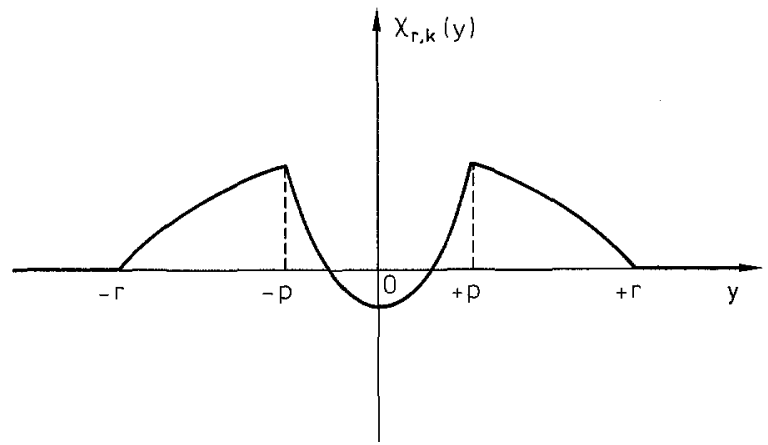

Fig. 1

Theorem 6. The function $\chi_{r, k}$ satisfies $\kappa^{*}\left(\chi_{r, k}\right)=k$ and minimizes $V_{1}(\chi, H)$ among all $\chi$ in $\mathscr{S}_{r}$ satisfying $\kappa^{*}(\chi) \leqq k$. Any other solution of this problem is equivalent to it.

This optimal solution is the counterpart of the tanh-estimator for location (Hampel, Rousseeuw and Ronchetti 1981). It satisfies the same optimality criterion, and (4.6) for $p \leqq|y| \leqq r$ looks similar to the expression of the redescending parts of the tanh-estimator. (This is not so surprising, because one can transform a scale problem into a location problem by means of the transformation $t=\ln |y|$.) An easier expression for these parts of $\chi_{r, k}$ is

$$
\chi_{r, k}(y)=\alpha \frac{\left(r^{2}\right)^{\beta}-\left(y^{2}\right)^{\beta}}{\left(r^{2}\right)^{\beta}+\left(y^{2}\right)^{\beta}} \quad \text { for } p \leqq|y| \leqq r
$$

where $\alpha=\left(A_{1}(k-1)\right)^{1 / 2}$ and $\beta=\frac{1}{2}\left((k-1) B_{1}^{2} / A_{1}\right)^{1 / 2}$.

Table 2 gives some values of the defining constants (which are unique by Theorem 6), as well as the efficiency $e=1 /\left(2 V_{1}\left(\chi_{r, k}, H\right)\right)=B_{1}^{2} /\left(2 A_{1}\right)$ (because the Fisher information at $\Phi$ equals 2), $\gamma_{u}^{*}\left(\chi_{r, k}\right)=\left(p^{2}-1+a\right) / B_{1}, \gamma_{s}^{*}\left(\chi_{r, k}\right)=\left(p^{2}-1\right.$ $+a) / \sqrt{A_{1}}$ and the local-shift sensitivity $\lambda^{*}=\sup _{y \neq t}\left|\chi_{r, k}(y)-\chi_{r, k}(t)\right| /\left(B_{1}|y-t|\right)$ (Hampel 1974). Note that the values of $e$ are typically smaller than those of the location case, which is a natural phenomenon: for example, compare $e \simeq 0.637$ of the median with $e \simeq 0.367$ of the MAD. The constants $A_{1}, B_{1}, a$ and $p$ were 
Table 2. Optimal Redescending $M$-estimators for Scale

\begin{tabular}{llllllllll}
\hline$c$ & $k$ & $A_{1}$ & $B_{1}$ & $p$ & $a$ & $e$ & $\gamma_{u}^{*}$ & $\gamma_{s}^{*}$ & $\lambda^{*}$ \\
\hline 3.0 & 6.0 & 0.167554 & 0.319908 & 1.079721 & 0.486660 & 0.3054 & 2.0395 & 1.5939 & 6.7502 \\
& 7.0 & 0.281931 & 0.465252 & 1.249943 & 0.393619 & 0.3839 & 2.0547 & 1.8004 & 5.3732 \\
& 8.0 & 0.391789 & 0.590050 & 1.381465 & 0.329294 & 0.4443 & 2.0977 & 1.9775 & 4.6825 \\
4.0 & 6.0 & 0.453990 & 0.709875 & 1.405979 & 0.293372 & 0.5550 & 1.7893 & 1.8851 & 3.9612 \\
& 7.0 & 0.620910 & 0.885645 & 1.560177 & 0.227110 & 0.6316 & 1.8758 & 2.1083 & 3.5233 \\
& 8.0 & 0.765560 & 1.026474 & 1.684394 & 0.181935 & 0.6882 & 1.9670 & 2.3076 & 3.2819 \\
5.0 & 6.0 & 0.637109 & 0.926499 & 1.549797 & 0.220007 & 0.6737 & 1.7505 & 2.0319 & 3.3455 \\
& 7.0 & 0.812012 & 1.095522 & 1.694232 & 0.167696 & 0.7390 & 1.8604 & 2.2618 & 3.0930 \\
& 8.0 & 0.957789 & 1.227069 & 1.812263 & 0.132274 & 0.7860 & 1.9694 & 2.4693 & 2.9538 \\
6.0 & 6.0 & 0.739162 & 1.038695 & 1.620538 & 0.187503 & 0.7298 & 1.7461 & 2.1095 & 3.1203 \\
& 7.0 & 0.909846 & 1.196082 & 1.757414 & 0.142829 & 0.7862 & 1.8655 & 2.3392 & 2.9386 \\
& 8.0 & 1.049618 & 1.317061 & 1.870063 & 0.112557 & 0.8263 & 1.9815 & 2.5473 & 2.8398 \\
7.0 & 6.0 & 0.797200 & 1.100010 & 1.658690 & 0.171035 & 0.7589 & 1.7475 & 2.1670 & 3.0158 \\
& 7.0 & 0.961937 & 1.247776 & 1.789971 & 0.130832 & 0.8093 & 1.8712 & 2.3806 & 2.8691 \\
& 8.0 & 1.095832 & 1.360908 & 1.898571 & 0.103467 & 0.8451 & 1.9899 & 2.5870 & 2.7902 \\
8.0 & 6.0 & 0.831873 & 1.135813 & 1.680886 & 0.161808 & 0.7754 & 1.7496 & 2.1788 & 2.9598 \\
& 7.0 & 0.991422 & 1.276475 & 1.808115 & 0.124388 & 0.8217 & 1.8752 & 2.4040 & 2.8330 \\
& 8.0 & 1.120784 & 1.384180 & 1.913823 & 0.098776 & 0.8547 & 1.9950 & 2.6084 & 2.7653 \\
\hline
\end{tabular}

computed iteratively. First $r$ and $k$ were read, together with some starting values. In the iteration, a new value of $p$ was found by applying NewtonRaphson to (4.7), and new values of $A_{1}, B_{1}$ and a were calculated by $\left(A_{1}\right)_{\text {new }}$ $=\left(\int \chi_{r, k}^{2} d \Phi\right)_{\text {old }},\left(B_{1}\right)_{\text {new }}=\left(\int \chi_{r, k}^{\prime} d \Phi\right)_{\text {old }}$ and $a_{\text {new }}=\left(a-\int \chi_{r, k} d \Phi\right)_{\text {old }}$ using Romberg integration. The algorithm continued until a precision of $\varepsilon=10^{-10}$ was reached, converging fast.

\section{Appendix}

Proof of Theorem 1. Assume that $\kappa_{u}^{*}:=\kappa_{u}^{*}(\eta)<\infty$. Let us suppose that there exists $\left(x_{0}, y_{0}\right)$ such that $\left|\eta\left(x_{0}, y_{0}\right)\right| \cdot\left\|M^{-1} x_{0}\right\|>\left[\left(\kappa_{u}^{*}-1\right) \operatorname{MSE}(\eta, H)\right]^{1 / 2}$. Without loss of generality, $y_{0} \notin D\left(x_{0}, \eta\right)$ and $y_{0}>0$. We also have $x_{0} \neq 0$, and we put $\psi(y):=\eta\left(x_{0}, y\right)\left\|M^{-1} x_{0}\right\|$ for all $y \notin C\left(x_{0}, \eta\right)$. When the factor $\left(M^{-1} x_{0}\right)^{t} Q M^{-1}\left(M^{-1} x_{0}\right)$ in (3.6) is strictly positive, we can repeat the proof of Theorem 1 of Rousseeuw (1981) which yields the desired contradiction. When this factor is negative or zero, a similar reasoning can be used (see Ronchetti and Rousseeuw 1982 for more details). When $\eta$ is monotone, $Q M^{-1} \geqq 0$ and $\gamma_{u}^{*}(\eta)<\infty$, (3.6) can only contain negative delta functions, so $\kappa_{u}^{*}(\eta) \leqq \sup (1$ $\left.+\eta^{2}(x, y)\left\|M^{-1} x\right\|^{2} / \operatorname{tr} V(\eta, H)\right) \leqq 1+\gamma_{u}^{*}(\eta)^{2} / \operatorname{MSE}(\eta, H)$.

Proof of Theorem 2. We have $M=E\left[y \eta(x, y) x x^{t}\right]$, so $I_{p}=E\left[y \eta(x, y) M^{-1} x x^{t}\right]$. Taking the trace,

$$
\begin{aligned}
p & =E\left[y \eta(x, y) x^{t} M^{-1} x\right] \leqq E\left[|y| \cdot|\eta(x, y)| \cdot\|x\| \cdot\left\|M^{-1} x\right\|\right] \leqq \gamma_{u}^{*}(\eta) E[|y| \cdot\|x\|] \\
& =\gamma_{u}^{*}(\eta) 2 \Phi^{\prime}(0) E[\|x\|],
\end{aligned}
$$


from which the desired inequality follows. If $E\left[x x^{t} /\|x\|\right]$ is a scalar matrix and $\eta(x, y)=\operatorname{sign}(y) /\|x\|$, then $M=2 \Phi^{\prime}(0) E\left[x x^{t} /\|x\|\right]=2 \Phi^{\prime}(0) E[\|x\|] \frac{1}{p} I_{p}$ so $\gamma_{u}^{*}(\eta)$ $=p(\pi / 2)^{1 / 2} / E[\|x\|]$.

Proof of Theorem 3. We have

$$
\begin{aligned}
\operatorname{MSE}(\eta, H) & =\operatorname{tr}\left(M^{-1} Q M^{-1}\right)=\operatorname{tr} E\left[M^{-1} \eta^{2}(x, y) x x^{t} M^{-1}\right] \\
& =\operatorname{tr} E\left[\left(\eta(x, y) M^{-1} x\right)\left(\eta(x, y) M^{-1} x\right)^{t}\right] \\
& =E\left[\left\{|\eta(x, y)| \cdot\left\|M^{-1} x\right\|\right\}^{2}\right] \leqq \gamma_{u}^{*}(\eta)^{2} .
\end{aligned}
$$

Making use of Theorem 1 , it follows that $\kappa_{u}^{*}(\eta) \geqq 1+\gamma_{u}^{*}(\eta)^{2} / \operatorname{MSE}(\eta, H) \geqq 2$. If $E\left[x x^{t} /\|x\|\right]$ is a scalar matrix and $\eta(x, y)=\operatorname{sign}(y) /\|x\|$, then $M$ $=2 \Phi^{\prime}(0) E[\|x\|] \frac{1}{p} I_{p}$ is a positive scalar matrix and $\operatorname{MSE}(\eta, H)=\gamma_{u}^{*}(\eta)^{2}$. As $Q M^{-1}=p(\pi / 2)^{1 / 2} E[\|x\|]^{-1} Q>0$ we can apply Theorem 1, yielding $\kappa_{u}^{*}(\eta)=1$ $+\gamma_{u}^{*}(\eta)^{2} / \operatorname{MSE}(\eta, H)=2$.

Proof of Lemma 1. Consider the function $\eta_{c, a}(x, y)=\psi_{c}(y a\|x\|) /(a\|x\|)$ for any $c>0$ and $a>0$. Because $p=1$ or $(R)$ holds and $\eta_{c, a}$ depends only on $x$ through $\|x\|$, we see that $M:=M\left(\eta_{c, a}\right)$ is a scalar matrix. In order to satisfy $M=\frac{1}{a} I_{p}$ it suffices to solve $\operatorname{tr}(M)=\frac{1}{a} \operatorname{tr}\left(I_{p}\right)$, yielding $\left.E[2 \Phi(c /(a\|x\|))-1)\|x\|^{2}\right]=p / a$. We put $f(a)=a E\left[(2 \Phi(c /(a\|x\|))-1)\|x\|^{2}\right]$, and try to solve $f(a)=p$. Now $f^{\prime}(a)$ $=E\left[\mid x \|^{2}\left\{2 \Phi(t)-1-2 t \Phi^{\prime}(t)\right\}\right]$ where $t=c /(a\|x\|)$, and because $2 \Phi(t)-1$ $=2 t \Phi^{\prime}(h)$ for some $0<h<t$ we have $f^{\prime}(a)>0$ for all $a>0$. Making use of $f(a)$ $=c E\left[\|x\| 2\{\Phi(t)-\Phi(0) / t]\right.$ we obtain $\lim _{a \downarrow 0} f(a)=0$ and $\lim _{a^{\uparrow} \infty} f(a)=2 \Phi^{\prime}(0) c E[\|x\|]$, so there is a (unique) solution of $f(a)=p$ if and only if $2 \Phi^{\prime}(0) c E[|x| \mid]>p$. We denote the resulting function $\eta_{c, a}$ by $\eta_{H K(c)}$. Now $M=M\left(\eta_{H K(c)}\right)=\frac{1}{a} I_{p}$, and $Q$ $=Q\left(\eta_{H K(c)}\right)$ is a positive scalar matrix, so $Q M^{-1}$ is also a positive scalar matrix. Therefore,

$$
\begin{aligned}
k(c):= & \kappa_{u}^{*}\left(\eta_{H K(c)}\right)=\sup \left\{1+\left(\psi_{c}^{2}(y a\|x\|)-2 \quad 1_{\{|y| a\|x\| \leqq c\}}\right.\right. \\
& \left.\left.\cdot(a x)^{t} Q M^{-1}(a x)\right) / \operatorname{MSE}\left(\eta_{H K(c)}, \mathrm{H}\right)\right\} \\
= & 1+c^{2} / \operatorname{MSE}\left(\eta_{H K(c)}, \mathrm{H}\right)<\infty .
\end{aligned}
$$

Because $\mathrm{a}$ is a continuous function of $c$ and $\operatorname{MSE}\left(\eta_{H K(c)}, H\right)=E\left[\psi_{c}^{2}(y a\|x\|)\right]$, the function $k$ is continuous on $(\alpha, \infty)$, where $\alpha=p(\pi / 2)^{1 / 2} / E[\|x\|]$. From Theorem 3 it follows that $k(c)>2$ for all $c$. Moreover, $\lim _{c \downarrow \alpha} k(c)=1+\alpha^{2} / \lim$ MSE $\left(\eta_{H K(c)}, H\right)=1+\alpha^{2} / \alpha^{2}=2$. When $c \uparrow \infty$, then it follows from Krasker (1980, proposition 1) that $M\left(\eta_{H K(c)}\right)=E\left[x x^{t}\right]=\frac{1}{p} E\left[\|x\|^{2}\right] I_{p}$, so $a \rightarrow p / E\left[\|x\|^{2}\right]$. Therefore, $E\left[\psi_{c}^{2}(y a\|x\|)\right] \rightarrow E\left[y^{2}\left(p / E\left[\|x\|^{2}\right]\right)^{2}\|x\|^{2}\right]=p^{2} / E\left[\|x\|^{2}\right]$, so $\underset{c \uparrow \infty}{\lim k(c)=1+\infty=\infty .}$ 
Proof of Theorem 5. The existence of $\eta_{H K(c)}$ follows from Lemma 1. Take any $\eta$ in $\mathscr{T}$ satisfying $\operatorname{MSE}(\eta, H)<\operatorname{MSE}\left(\eta_{H K(c)}, H\right)$. Then $\gamma_{u}^{*}(\eta)>\gamma_{u}^{*}\left(\eta_{H K(c)}\right)$ by Theorem 4. From Theorem 1 it follows that $\kappa_{u}^{*}(\eta) \geqq 1+\gamma_{u}^{*}(\eta)^{2} / \operatorname{MSE}(\eta, H)>1$ $+\gamma_{u}^{*}\left(\eta_{H K(c)}\right)^{2} / \mathrm{MSE}\left(\eta_{H K(c)}, H\right)$. The latter expression equals $\kappa_{u}^{*}\left(\eta_{H K(c)}\right)$ by Theorem 1 because $Q M^{-1} \geqq 0$ from the proof of Lemma 1 , hence $\kappa_{u}^{*}(\eta)>\kappa_{u}^{*}\left(\eta_{H K(c)}\right)$. This proves the optimality of $\eta_{H K(c)}$. Now take another function $\tilde{\eta}$ minimizing $\operatorname{MSE}(\eta, H)$ subject to $\kappa_{u}^{*}(\eta) \leqq k$. But then $\operatorname{MSE}(\tilde{\eta}, H)=\operatorname{MSE}\left(\eta_{H K(c)}, H\right)$ and

$$
\gamma_{u}^{*}(\tilde{\eta}) \leqq\left[\left(\kappa_{u}^{*}(\tilde{\eta})-1\right) \operatorname{MSE}(\tilde{\eta}, H)\right]^{1 / 2} \leqq\left[(k-1) \operatorname{MSE}\left(\eta_{H K(c)}, H\right)\right]^{1 / 2}=c
$$

so $\tilde{\eta}$ is equivalent to $\eta_{H K(s)}$ by Theorem 4 .

Proof of Theorem $1^{\prime}$. Assume that $\kappa_{s}^{*}(\eta)<\infty$ and that there exists $\left(x_{0}, y_{0}\right)$ such that $\left|\eta\left(x_{0}, y_{0}\right)\right|\left(x_{0}^{t} Q^{-1} x_{0}\right)^{1 / 2}>\left(\kappa_{s}^{*}(\eta)-p\right)^{1 / 2}$ where $y_{0} \notin D\left(x_{0}, \eta\right)$ and $y_{0}>0$. The proof then follows the same lines as that of Theorem 1, but now $\psi(y)$ $:=\eta\left(x_{0}, y\right)\left(x_{0}^{t} Q^{-1} x_{0}\right)^{1 / 2}$.

Proof of Theorem $2^{\prime}$. We have $Q=E\left[\eta^{2}(x, y) x x^{t}\right]$, so $I_{p}=E\left[\eta^{2}(x, y) x x^{t} Q^{-1}\right]$. Taking traces,

$$
p=E\left[\eta^{2}(x, y) x^{t} Q^{-1} x\right]=E\left[\left(|\eta(x, y)|\left(x^{t} Q^{-1} x\right)^{1 / 2}\right)^{2}\right] \leqq \gamma_{s}^{*}(\eta)^{2} .
$$

If $E\left[x x^{t} /\|x\|^{2}\right]$ is a scalar, then $\eta(x, y)=\operatorname{sign}(y) /\|x\|$ satisfies $Q=E\left[x x^{t} /\|x\|^{2}\right]$ $=\frac{1}{p} I_{p}$ (because its trace equals 1), hence $\gamma_{s}^{*}(\eta)=\sup |\eta(x, y)|\left(x^{t} Q^{-1} x\right)$ $=\sup \frac{1}{\|x\|}\|x\| \sqrt{p}=\sqrt{p}$.

Proof of Theorem 3'. From Theorems $1^{\prime}$ and $2^{\prime}$, it follows that $\kappa_{s}^{*}(\eta) \geqq p$ $+\gamma_{s}^{*}(\eta)^{2} \geqq p+(\sqrt{p})^{2}=2 p$. If $E\left[x x^{t} /\|x\|^{2}\right]$ is a scalar, then $\eta(x, y)=\operatorname{sign}(y) /\|x\|$ satisfies $\gamma_{s}^{*}(\eta)=\sqrt{\mathrm{p}}$ by Theorem $2^{\prime}$. Moreover $M=2 \Phi^{\prime}(0) \cdot E\left[x x^{t} /\|x\|\right]>0$, so $\kappa_{s}^{*}(\eta)=p+\gamma_{s}^{*}(\eta)^{2}=2 p$ by Theorem $1^{\prime}$.

Proof of Lemma $1^{\prime}$. The existence of $\eta_{K W(c)}$ for $c>\sqrt{p}$ follows from Krasker and Welsch (1982, Theorem 2) or Stahel (1981, p. 28); the "only if" part is a consequence of 'Theorem 2 ' above. It holds that

$$
\gamma_{s}^{*}\left(\eta_{\mathrm{KW}(\mathrm{c})}\right)=\sup \left\{\left|\psi_{c}(y\|A x\|)\right|\left(x^{t} A^{t} A x\right)^{1 / 2} /\|A x\|\right\}=\sup \{c\|A x\| /\|A x\|\}=c .
$$

Moreover, $M\left(\eta_{K W(c)}\right)>0$ because for all $z \neq 0$ it holds that

$$
z^{t} M\left(\eta_{K W(c)}\right) z=E\left[\eta_{K W(c)}^{\prime}(x, y) z^{t} x x^{t} z\right]=E\left[(2 \Phi(c /\|A x\|)-1)\left(z^{t} x\right)^{2}\right]>0 .
$$

From Theorem 1'

$$
\kappa_{s}^{*}\left(\eta_{K W(c)}\right)=p+\gamma_{s}^{*}\left(\eta_{K W(c)}\right)^{2}=p+c^{2} .
$$

Proof of Theorem 5'. Analogous to Theorem 5, making use of Theorems $1^{\prime}$ and $4^{\prime}$.

Proof of Theorem 6. We first show that $\kappa^{*}\left(\chi_{r, k}\right)=k$. On $(p, r)$ and $(-r,-p)$ it holds that $1+\chi_{r, k}^{2}(y) / A_{1}-2 y \chi_{r, k}^{\prime}(y) / B_{1} \equiv k$. For $|y|>r$ this expression reduces to 
1 which is smaller than $k$ because $k \geqq 1+(a-1)^{2} / A_{1}$. Because $y^{2}-1+a$ is monotone for $y \geqq 0$, positive in $p$ and negative in 0 , there exists a number $b$ in $(0, p)$ satisfying $b^{2}-1+a=0$. On $[-b, b]$ it holds that $1+\chi_{r, k}^{2}(y) / A_{1}$ $-2 y \chi_{r, k}^{\prime}(y) / B_{1} \leqq 1+\left(y^{2}-1+a\right) / A_{1} \leqq 1+(a-1)^{2} / A_{1} \leqq k$. (The condition $1+(a$ $-1)^{2} / A_{1} \leqq k$ is also necessary, because otherwise $\kappa^{*}\left(\chi_{r, k}\right) \geqq 1+\chi_{r, k}^{2}(0) / A_{1}>k$.) On $[b, p)$ and $(-p,-b]$ it holds that $1+\chi_{r, k}^{2}(y) / A_{1}-2 y \chi_{r, k}^{\prime}(y) / B_{1} \leqq 1+\left(p^{2}-1\right.$ $+a)^{2} / A_{1} \leqq 1+A_{1}(k-1) / A_{1}=k$, making use of $(4.9)$ and the fact that the square of a hyperbolic tangent is always smaller than 1 . For the optimality, it suffices to show that $\chi_{r, k}$ minimizes $\int \chi^{2} d \Phi$ subject to $\chi \in \mathscr{S}_{r}, \int z \chi^{\prime}(z) d \Phi(z)=B_{1}$ and $\kappa^{*}(\chi) \leqq k$. It holds that $\int\left(y^{2}-1+a-\chi(y)\right)^{2} d \Phi(y)=\int\left(y^{2}-1+a\right)^{2} d \Phi(y)-2 B_{1}$ $+\int \chi^{2} d \Phi$, so it suffices to minimize the left member of this inequality. The rest of the proof is analogous to Theorem 4.1 of (Hampel, Rousseeuw and Ronchetti 1981).

Acknowledgments. This work contains parts of the first author's $\mathrm{Ph}$. $\mathrm{D}$. dissertation, which was written under the generous guidance of Prof. F. Hampel. The research of the second author was supported by the Belgian National Science Foundation. The authors are grateful to Prof. I. Olkin, Prof. C. Field and Prof. A. Samarov for their valuable suggestions.

\section{References}

Bickel, P.J.: One-step Huber estimates in the linear model. J. Amer. Statist. Assoc. 70, 428-434 (1975)

Collins, J.R.: Robust estimation of a location parameter in the presence of asymmetry. Ann. Statist. 4, 68-85 (1976)

Donoho, D.L., Huber, P.J.: The notion of breakdown point. In: Festschrift for E.L. Lehmann, Wadsworth (1983)

Hampel, F.R.: Robust estimation: a condensed partial survey. Z. Wahrscheinlichkeitstheorie verw. Gebiete 27, 87-104 (1973)

Hampel, F.R.: The influence curve and its role in robust estimation. J. Amer. Statist. Assoc. 69. 383-393 (1974)

Hampel, F.R.: Optimally bounding the gross-error-sensitivity and the influence of position in factor space. Proceedings of the ASA Statistical Computing Section, pp. 59-64, 1978

Hampel, F.R., Rousseeuw, P.J., Ronchetti, E.: The change-of-variance curve and optimal redescending $M$-estimators. J. Amer. Statist. Assoc. 76, 643-648 (1981)

Handschin, E., Kohlas, J., Fiechter, A., Schweppe, F.: Bad data analysis for power system state estimation. IEEE Trans. Power Apparatus Syst. 2, 329-337 (1975)

Hill, R.W.: Robust regression when there are outliers in the carriers. Unpublished Ph.D. dissertation, Harvard University (1977)

Holland, P.W., Welsch, R.E.: Robust regression using iteratively reweighted least squares. Commun. Statist. A 6, 813-827 (1977)

Huber, P.J.: Robust estimation of a location parameter. Ann. Math. Statist. 35, 73-101 (1964)

Huber, P.J.: Robust regression: asymptotics, conjectures and Monte Carlo. Ann. Statist. 1, 799-821 (1973)

Huber, P.J.: Robust Statistics. New York: Wiley 1981

Krasker, W.S.: Estimation in linear regression models with disparate data points. Econometrica 48 , $1333-1346(1980)$

Krasker, W.S., Welsch, R.E.: Efficient bounded-influence regression estimation. J. Amer. Statist. Assoc. 77, 595-604 (1982)

Lambert, D.: Influence functions for testing. J. Amer. Statist. Assoc. 76, 649-657 (1981)

Mallows, C.L.: On some topics in robustness. Unpublished memorandum. Murray Hill, New Jersey: Bell Telephone Laboratories 1975 
Marazzi, A.: Robust linear regression programs in ROBETH. Research Report No. 23, Fachgruppe für Statistik, ETH Zürich (1980)

Maronna, R.A., Yohai, V.J.: Asymptotic behaviour of general $M$-estimates for regression and scale with random carriers. Z. Wahrscheinlichkeitstheorie verw. Gebiete 58, 7-20 (1981)

Ronchetti, E.: Robustheitseigenschaften von Tests. Diploma thesis, ETH, Zürich (1979)

Ronchetti, E.: Robust alternatives to the F-test for the linear model. In: Probability and Statistical Inference. W. Grossmann, C. Pflug, W. Wertz (eds.), pp. 329-342. Dortrecht: Reidel 1982a

Ronchetti, E.: Robust testing in linear models: the infinitesimal approach. Ph.D. thesis, ETH, Zürich (1982b)

Ronchetti, E., Rousseeuw, P.J.: A robust $F$-test for the linear model. 13th European Meeting of Statisticians, Brighton, Abstracts Book, pp. 210-211, 1980

Ronchetti, E., Rousseeuw, P.J.: Change-of-variance sensitivities in regression analysis. Research Report No. 36. Fachgruppe für Statistik, ETH Zürich (1982)

Rousseeuw, P.J.: A new infinitesimal approach to robust estimation. Z. Wahrscheinlichkeitstheorie verw. Gebiete 56, 127-132 (1981)

Rousseeuw, P.J.: Most robust $M$-estimators in the infinitesimal sense. Z. Wahrscheinlichkeitstheorie verw. Gebiete 61, 541-551 (1.982a)

Rousseeuw, P.J.: Least median of squares regression. Research Report No. 178, Centrum voor Statistiek en Operationeel Onderzoek, VUB Brussels (1982b). To appear in J. Amer. Statist. Assoc.

Rousseeuw, P.J., Ronchetti, E.: The influence curve for tests. Research Report No. 21, Fachgruppe für Statistik, ETH Zürich (1979)

Rousseeuw, P.J., Ronchetti, E.: Influence curves for general statistics. J. Comput. Appl. Math. 7, 162-166 (1981)

Ruppert, D., Carroll, R.J.: Trimmed least squares estimation in the linear model. J. Amer. Statist. Assoc. 75, 828-838 (1980)

Schrader, R.M., Hettmansperger, T.P.: Robust analysis of variance based upon a likelihood ratio criterion. Biometrika 67, 93-101 (1980)

Schrader, R.M., McKean, J.W.: Robust analysis of variance. Commun. Statist. A 6, 879-894 (1977)

Siegel, A.F.: Robust regression using repeated medians. Biometrika 69, 242-244 (1982)

Stahel, W.A.: Robuste Schätzungen: infinitesimale Optimalität und Schätzungen von Kovarianzmatrizen. Ph.D. thesis, ETH, Zürich (1981)

Received November 7, 1983 